\title{
Australia's National Health Programs: An Ontological Mapping
}

\author{
Arkalgud Ramaprasad \\ University of Illinois at Chicago \\ Khin Than Win \\ University of Wollongong \\ win@uow.edu.au
}

Thant Syn

Texas A\&M International University

\section{Ghassan Beydoun}

University of Technology, Sydney,

\author{
Linda Dawson \\ University of Wollongong,
}

\section{Abstract}

Australia has a large number of health program initiatives whose comprehensive assessment will help refine and redefine priorities by highlighting areas of emphasis, under-emphasis, and non-emphasis. The objectives of our research are to: (a) systematically map all the programs onto an ontological framework, and (b) systemically analyse their relative emphases at different levels of granularity. We mapped all the health program initiatives onto an ontology with five dimensions, namely: (a) Policy-scope, (b) Policy-focus, (c) Outcomes, (d) Type of care, and (e) Population served. Each dimension is expanded into a taxonomy of its constituent elements. Each combination of elements from the five dimensions is a possible policy initiative component. There are 30,030 possible components encapsulated in the ontology. It includes, for example: (a) National financial policies on accessibility of preventive care for family, and (b) Local-urban regulatory policies on cost of palliative care for individual-aged. Four of the authors mapped all of Australia's health programs and initiatives on to the ontology. Visualizations of the data are used to highlight the relative emphases in the program initiatives. The dominant emphasis of the program initiatives is: [National] [educational, personnelphysician, information] policies on [accessibility, quality] of [preventive, wellness] care for the [community]. However, although (a) information is emphasized technology is not; and (b) accessibility and quality are emphasized cost, satisfaction, and quality are not. The ontology and the results of the mapping can help systematically reassess and redirect the relative emphases of the programs and initiatives from a systemic perspective.

Keywords: ontology; health initiatives; health program; ontological framework

\section{Introduction}

Health programs promote, restore and maintain health (World Health Organization 2011). National health programs vary significantly across countries based on their needs, initiatives, policies and stakeholders involved (Núñez Mondaca, Ramaprasad, and Syn 2015, Ramaprasad, Nanda Kumar, and Syn 2015). They evolve with the changing environment, demographics, and the requirements of the country's population. Australia's Department of Health has several programs and initiatives to support the nation's health system. Its states, territories, and local governments have their own programs which operate concurrently. We focus on Australia's national programs and initiatives.

Health in All Policies—one of the outcomes of the 1986 Ottawa Charter for Health Promotionintroduced the concept of better health, improved population health outcomes, and closing the health gap (Kickbusch, McCann, and Sherbon 2008) through policies in all sectors and areas. Nations all over the world have been seeking ways to implement this. In the USA, Healthy 
People 2020 initiatives built on the social determinants of health approach (Koh et al. 2011). In Australia, policies across different health sectors recognise the social determinants of the health approach too (Fisher et al. 2016).

Government policy and health planning set the direction for the distribution of health services, workforce, resource allocation, and social environment (Heilbrunn-Lang et al. 2016). Health service needs of the population have to be satisfied by the healthcare workforce (Lopes, Almeida, and Almada-Lobo 2015). Healthcare disparities or inequalities exist in different health communities. A culturally responsive framework was proposed in 2012 for the culturally diverse population in Australia (Gill and Babacan 2012) to provide (a) better links between access, equity, quality, and safety, (b) better health outcomes for culturally and linguistically diverse (CALD) populations, (c) enhanced cost effectiveness of service provision, and (d) culturally responsive and existing standards compliant services (Seeleman et al. 2015).

Accessibility to health services, workforce distribution, recruiting and retaining staff, and having quality care services have been the challenges for rural Australia (Malatzky and Bourke 2016, Bourke et al. 2012). Support in workforce, training for rural and regional healthcare, continuing professional development access, family support, recognition, and remuneration have been identified as needs for the workforce in Australia (Larkins and Evans 2014).

Australian National Health Policy investment and deinvestment timeline was presented by Smith, Crawford, and Signal (2016). Release of National Preventive Health Strategy in 2008 and National Primary Healthcare Strategic Framework in 2013 are noteworthy while the deletion of Australian National Preventive Health Agency and the cessation of the National Partnership Agreement on Preventive Health in 2014 are of particular concern (Smith, Crawford, and Signal 2016). Significant health policy changes under government policy changes or budget will have impact on health outcome (Moore and Yeatman 2014). In this context, we analyse Australia's national health programs systematically and systemically, at a point in time. The analysis provides the 'big picture' of the areas of strong, moderate, and no emphasis in the programs. As such, it highlights the strengths and weaknesses of the programs and provides a roadmap for changing the future trajectory.

Australia's Department of Health's vision, purpose, and strategic priorities are as follows (Australian Government Department of Health 2016c):

Vision: Better health and wellbeing for all Australians, now and for future generations

Purpose: Lead and shape Australia's health system and sporting outcomes through evidence based policy, well targeted programs, and best practice regulation

\section{Strategic Priorities:}

Better health outcomes and reduced inequality through:

- $\quad$ An integrated approach that balances prevention, primary, secondary, and tertiary care

- $\quad$ Promoting greater engagement of individuals in their health and healthcare

- $\quad$ Enabling access for the most disadvantaged including Aboriginal and Torres Strait Islander people, people in rural and remote areas and people experiencing socio-economic disadvantage

Affordable, accessible, efficient, and high quality health system through:

- $\quad$ Partnering and collaborating with others to deliver health programs

- $\quad$ Better, more cost-effective patient care through innovation and technology

- Regulation that protects the health and safety of the community

Better sport outcomes through:

- Boosting participation opportunities for all Australians 
- $\quad$ Optimising international performance

- $\quad$ Safeguarding integrity in sport

To fulfil the above aspiration, the Department has a large number of programs and initiatives. The current list is available at Australian Government Department of Health (2016d). In April 2015 when this study was conducted, there were 163 programs listed on the site. We posed the question: Do the programs and initiatives fit the aspiration? The feedback from this study will help answer this question and aid future program redirection and development.

Health programs are formulated over long periods of time, by different governments, and different agencies of the governments. They may originate in legislation passed in the parliament, the reports of committees, thejudgment of courts, and response to public pressure. Sometimes the programs are products of systematic thinking about a problem by a committee, think-tank, or other entities. Often they are formulated opportunistically to respond to the needs of the time, and incrementally to change or correct programs of the past. Australia's Department of Health was established in 1921-its name, structure, and function have been changed a number of times since then (Australian Government Department of Health 2016b).

The annual reports of the department summarize the performance in terms of a variety of outcome measures such as: (a) Population Health, (b) Access to Pharmaceutical Services, (c) Access to Medical and Dental Services, (d) Acute Care, (e) Primary Health Care, (f) Private Health, (g) Health Infrastructure, Regulation, Safety and Quality, (h) Health Workforce Capacity, (i) Biosecurity and Emergency Response, and (j) Sport and Recreation.

Comprehensive assessment including an understanding of interactions and relationships of a country's health programs and initiatives will help refine and redefine priorities by highlighting areas of emphasis, under-emphasis, and non-emphasis. Without an appropriate means to grasp the 'big picture' of the entire nation's healthcare policies, policy-makers play the risk of missing the forest for the trees. Bennett and Peters $(2015,10)$ suggest four reasons for such an assessment:

1. To motivate health system reforms;

2. To promote harmonization and alignment across actors in the health system;

3. To translate health systems reforms into meaningful tracking and evaluation of systems performance; and

4. To facilitate learning through cross-country comparisons.

The description of Australia's programs and initiatives constitutes a large volume of text data. There are few techniques to systematically and systemically assess the programs based on this 'big text data'. In this paper we use meta-analysis and synthesis of the data using an ontology as a tool to mine the data (Ramaprasad and Syn 2015). The ontology provides a set of explanatory terms and relations that anchor the description of the domain enabling consensus and explicit sharing of reflections on Australia's health programs and initiatives. We will illustrate its use to map the topography of this knowledge domain and visualize the "knowledge structure" (Zhang et al. 2012) of the domain. The health programs and initiatives are treated as realizations of the concepts articulated in the ontology. They provide textual empirical data that sample the ontological topography. The topography represents a "virtual knowledge landscape" (Scharnhorst 2001, 505) based on textual empirical data. It will help to visually recognize the coherence and lack of it in the cumulative domain knowledge, and therefore help correct the lacuna when appropriate (Hoeffner and Smiraglia 2013, Noar and Zimmerman 2005). It will help visualize the bright, light, and blank/ blind spots in the knowledge domain. Thus, it will provide "support for navigating the knowledge landscape." (Kazimierczak et al. 2012 , 1) Further, "[i]ncrementally computed information landscapes are an effective means to visualize longitudinal changes in large document repositories..." (Syed et al. 2012, 352).

"Given diverse rationales for undertaking HSAs [Health System Assessments], there is no one standardized framework to guide them. Frameworks and tools should be selected to reflect 
proposed use..." (Bennett and Peters 2015, 9). In the following, we present an ontology-based assessment of Australia's health programs and initiatives. It is adapted from an earlier effort to analyse and synthesize the healthcare policies of Chile (Núñez Mondaca, Ramaprasad, and Syn 2015). We will describe the deconstruction of the construct of 'healthcare policy' into its constituent dimensions, and the dimensions into their constituent elements. We will then present the mapping of Australia's national health programs and initiatives onto the ontology to illuminate the 'bright', 'light', and 'blind/blank' spots which indicate the policy areas with significant emphasis, little emphasis, and no emphasis, respectively. Lastly, we will discuss how the ontological framework can be used to articulate the key themes and questions systematically and systemically. Such a meta-analysis of the health programs in their entirety will help develop a roadmap for the future.

\section{Ontology of Health Policies}

The ontology of healthcare policy represents our conceptualization of the healthcare policy domain (Gruber 2008). It is an "explicit specification of [our] conceptualization," (Gruber 1995, 908) and can be used to systematize the description of a complex system (Cimino 2006) such as national healthcare policies. The ontology organizes the terminologies and taxonomies that constitute healthcare policies. "Our acceptance of [the] ontology is... similar in principle to our acceptance of a scientific theory, say a system of physics; we adopt, at least insofar as we are reasonable, the simplest conceptual scheme into which the disordered fragments of raw experience can be fitted and arranged." (Quine 1961, 16)

The method of constructing and presenting an ontology for a domain is explained in greater detail in Ramaprasad and Syn (2014a, 2014b). It is an iterative process that systemically and systematically deconstructs healthcare policy into its dimensions and associated elements. It is a new method of analysing and synthesizing knowledge in a domain (Ramaprasad and Syn 2015, Tate et al. 2015). It has been applied to analysing and synthesizing Chile's and India's national healthcare policies (Núñez Mondaca, Ramaprasad, and Syn 2015, Ramaprasad, Nanda Kumar, and Syn 2015). Here we apply it to policies governing Australia's healthcare system.

The challenge is to construct an ontology which is a logical, parsimonious, and complete description of Australia's national healthcare policy. It has to be logical in the deconstruction of the domain, and parsimonious yet complete in the representation of the domain. It has to be a closed description of a country's healthcare policy in its entirety yet adaptable to changes in the domain.

There can be multiple points of views to study a complex domain like national healthcare policy. Each view can be represented in its own ontology. It is a 'wicked' problem (Churchman 1967) with many potential formulations (Ramaprasad and Syn 2014a). Each ontology can be seen as a lens to study a country's healthcare policy.

The ontological analysis provides a holistic approach to understanding healthcare policies; the traditional methods tend to be linear focusing on a subset of its elements, fragments, or components. In fact, our method simply reorganizes the traditional bodies of knowledge derived from traditional methods in a novel way to provide a holistic understanding. Thus, dimension and taxonomies constituting the ontology are not new; most of them are in the literature on healthcare, public policy, and development. The ontology helps synthesize the knowledge from these disciplines in the context of healthcare policies to provide a holistic perspective.

\subsection{Construction of the Ontology}

The domain of interest is healthcare policy. It can be conceptualized as a composite of two subdomains-healthcare' and 'policy' - each with a considerable corpus of research and practice but in different disciplines. Each subdomain can be further divided as discussed below. 
The construct of healthcare, especially in the context of healthcare policy, commonly refers to: (a) a population whose health is of concern to the policy makers; (b) the different types of care which constitute healthcare; and (c) the various outcomes desired of healthcare. The distinction by population is made to recognize their different needs, to cater to them effectively. Some needs have to be addressed at the individual level; some may be addressed at the family or community level. Some needs are very specific (for example, pre-natal children); some are more general (for example, adults). The distinction by type of care is necessary to accommodate the different types, amount, and cost associated with each type. Healthcare policies have to account for these differences to be effective. Healthcare policies seek many outcomes from the healthcare of the population. These outcomes are specified in the design of the policies, measured to assess them, and used to provide feedback to (re)direct their trajectory. Healthcare policies seek multiple outcomes for the target population based on the different types of care.

Policies have many attributes. Among them, their scopeand focus directly impact the outcomes of different types of care for the various populations. Healthcare is a subject that is concurrently addressed at the global, national, local, and institutional levels. Although the subject of our study is Australia's national healthcare policies, some of their scope may bemore general, specific, or both. The scope can affect the breadth and depth of a policies effects on the health outcomes.

Simultaneously with the scope (which is the equivalent of geographical focus) the substantive focus of the policies varies. Policies seek to focus on the key attributes of the system necessary to deliver care to the population. Some policies may have a singular focus and some may have multiple foci. In conjunction with their scope the focus affects the outcomes.

Thus, we deconstruct healthcare policy into (a) the scope of the policy, (b) the focus of the policy, (c) the desired outcomes of the policy, (d) the type of care which is the objects of the policy, and (e) the population for which it is intended. A healthcare policy is ideally seen as having a defined scope, being focused on an aspect of healthcare, with desired outcomes, for particular types of care, targeted at a given population. The underlying argument is:

Healthcare policy $=$ Scope + Focus + Outcome + Care + Population

These five dimensions define the domain of healthcare policies. In practice, all healthcare policies may not cover all the dimensions. For example, some may not specify the outcome, some the focus, and some a combination of these. We will refer to policies in which some dimensions are excluded from the definition as fragments of policies rather than full policies.

Each dimension of the ontology is expressed by a taxonomy of its constituent elements (Figure 1). The elements are defined in the glossary in Appendix. The taxonomies are derived from the common terminology in the body of knowledge on each dimension, especially in the healthcare policy domain. (Note: We will capitalize the words which refer to the dimensions and elements in the ontology, except in describing full or partial components.)

Thus, the Scope of a policy may be Global, National, Local-Urban, Local-Rural, or restricted to the Provider. They are primarily defined geographically. The categories are arranged from the most general to the specific and are grounded in practice in Australia and other countries (Chile, India, and China) that have been studied by two of the authors (Núñez Mondaca, Ramaprasad, and Syn 2015, Ramaprasad, Nanda Kumar, and Syn 2015). The research literature addresses these levels but does not present it as a taxonomy. They evolved iteratively through abstraction from the corpus of policies and application to the same corpus to test the fit. The last category, Provider, for example, was added to accommodate the distinctive policies of these entities which are not inherited from the different levels (of the Government) above. 


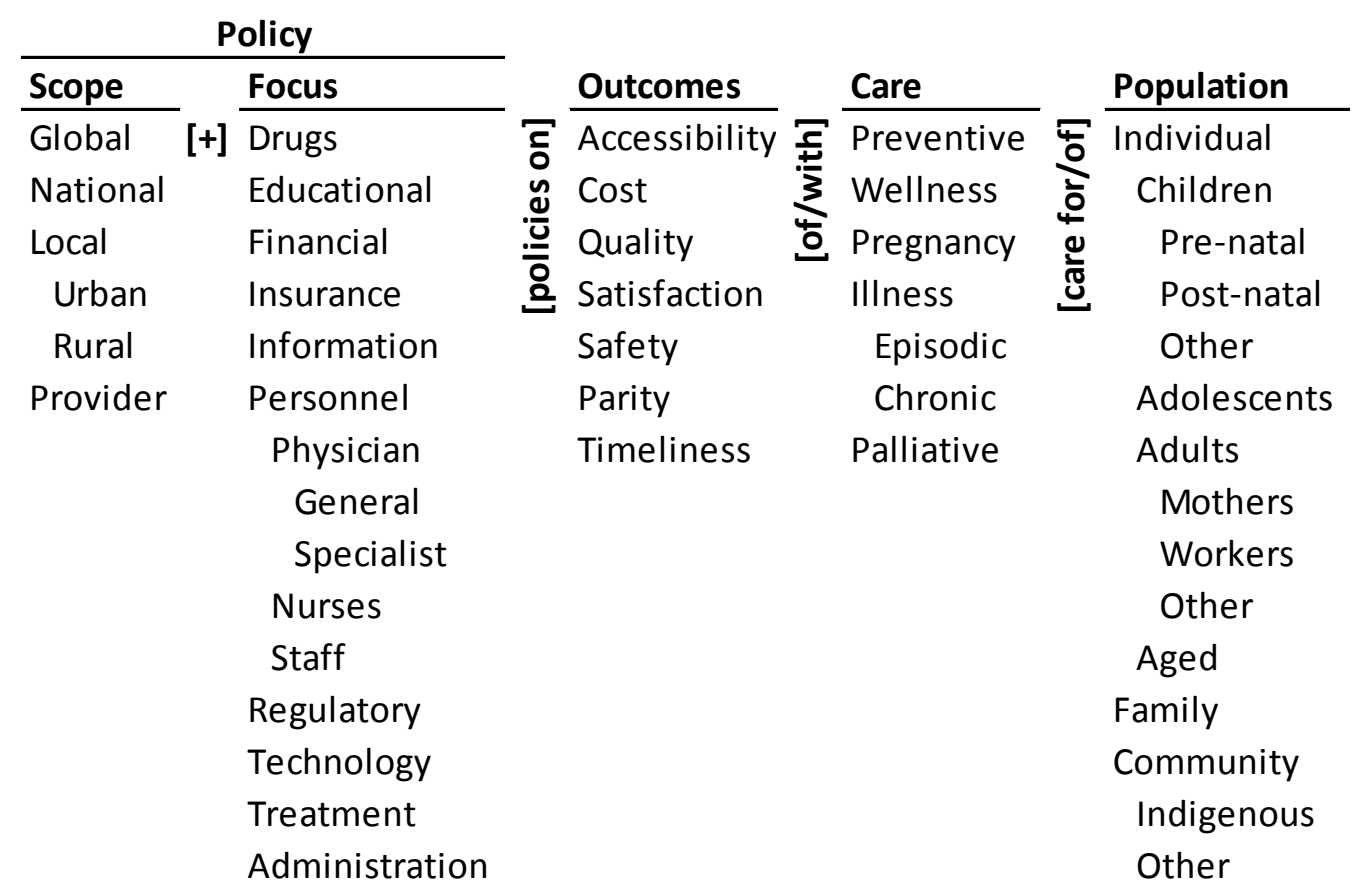

Illustrative components (total $=5 * 13 * 7 * 6 * 11=30,030$ ):

National financial policies on accessibility of preventive care for family. Local $_{\text {urban }}$ regulatory policies on cost of palliative care for individual ${ }_{\text {aged. }}$. Provider administration policies on cost of illness episodic $_{\text {care of individual }}$ adolescents.

Figure 1 Ontology of National Healthcare Policy with Illustrative Components

The Scope categories represent the units of attention and action of the policies-the levels at which policies address the healthcare of a population. They reflect the porosity of geographical boundaries in healthcare, especially in the context of air travel and fast spreading epidemics, making it necessary to address the issues at all levels simultaneously. They also reflect the particularity of many health problems which have to be addressed locally. Urban health problems, for example, are often different from rural ones. At thelowest level of the dimension, the Provider's policies may ultimately determine the care delivered and its outcome. Thus:

Scope $\subset$ [Global, National, Local (Urban, Rural), Provider]

The Focus of the Policy may be Drugs, Educational, Financial, Insurance, Information, Personnel, Regulatory, Technology, Treatment, or Administration. The Personnel focus may be on the Physician, Nurses, or Staff. These can be said to be the common instruments of healthcare policy to achieve the outcomes. They are not presented in any particular order. The categories are grounded in literature (Bourke et al. 2012, Larkins and Evans 2014, Malatzky and Bourke 2016) and practice, derived iteratively through abstraction from and application to the corpus. They cover all the primary foci of healthcare policies. A policy may have a singular focus or multiple foci. However, a combination of these foci/instruments is essential for the delivery of care for different populations to achieve the outcomes. The emphases on these foci may vary significantly based on the type of care, the population, and the desired outcomes. Drugs, for example, may be important for Illness care; on the other hand, Education may be important for Preventive care. Thus:

Focus $\subset$ [Drugs, Educational, Financial, Insurance, Information, Personnel, Regulatory, Technology, Treatment, Administration]

Personnel $\subset$ [Physician (General, Specialist), Nurses, Staff] 
The desired Outcomes of a healthcare policy have been articulated by the US Government (Ramaprasad, Syn, and Thirumalai 2015), the WHO (World Health Organization), and in the policies themselves. Collating and combining them, the Outcomes of the Policy may be Accessibility, Cost, Quality, Satisfaction, Safety, Parity, or Timeliness of healthcare. These categories comprehensively cover the desired outcomes of the policies. They are not presented in any particular order; Accessibility however appears to be the primary concern universally. Understandably, without accessibility the other outcomes would not be relevant.

Outcomes $\subset$ [Accessibility, Cost, Quality, Satisfaction, Safety, Parity, Timeliness]

The types of healthcare are fairly well established, although they may not be presented as a formal taxonomy. Historically, healthcare has been synonymous with Illness care; Preventive, Wellness, and Palliative care are of recent origin. Within Illness care, Chronic care become a distinctive subcategory with the increasing prevalence of chronic illnesses such as diabetes, and even some types of cancers being designated as Chronic conditions. Pregnancy care is defined as a separate category with the increasing focus on maternal and child health and reproductive health. Thus, the Care could be Preventive, for Wellness, Pregnancy, Illness (Episodic or Chronic), or Palliative.

Care $\subset$ [Preventive, Wellness, Pregnancy, Illness (Episodic, Chronic), Palliative]

Last, Population cared for may be the Individual, Family, or Community. The Individuals may be Children (Pre-natal or Post-natal), Adolescents, Adults (Mothers, Workers), and Aged. (Gill and Babacan 2012, Heilbrunn-Lang et al. 2016, Lopes, Almeida, and Almada-Lobo 2015) The sub-categories and the sub-sub-categories in the Population taxonomy indicate the finegrained attention to these groups in the policies based on their healthcare needs. They are grounded in the policies themselves and extracted through the iterative process of abstraction and application described earlier. For example, Australia's health programs pay special attention to Indigenous communities. In contrast, Chile and India's policies (Núñez Mondaca, Ramaprasad, and Syn 2015, Ramaprasad, Nanda Kumar, and Syn 2015) do not highlight their indigenous (tribal in India) populations. Hence they form a separate subcategory. Thus:

Population $\subset$ [Individual, Family, Community]

Individual $\subset$ [Children (Pre-natal, Post-natal, Other), Adolescents, Adults (Mothers, Workers, Other), Aged]

\section{Community $\subset$ [Indigenous, Other]}

Thus, the ontology is a product of: (a) logical decomposition and recomposition of the domain, (b) abstraction from and application to the policies in the domain, and (c) appropriation and adaptation of taxonomies from the research and policy corpus of the domain. It is a systemic and systematic representation of the domain. In the next section, we discuss how the ontology can be used as a lens to map the domain and study it.

\section{2 'Bright', 'Light', 'Blind/Blank' Spots}

The ontology in Figure 1 helps visualize the dimensions, sub-dimensions, elements, fragments, and components of healthcare policy in natural English. Each dimension is represented by a column. The taxonomy of elements constituting each dimension/ sub-dimension is listed in the respective column. All the elements together constitute the elements of the system of policies. The columns are connected by adjacent words/ phrases - they help concatenate the elements from different columns into phrases/sentences which describe the fragments/components which constitute the system.

Thus, for example: (a) accessibility of preventive care for family can be a fragment of a system, and (b) local-urban regulatory policies on cost of palliative care for individual-aged can be a component of a system. There are $\left(5^{*} 13^{*} 7 * 6 * 11=30,030\right)$ potential first-order components of the system of policies encapsulated in the ontology. If one considers interactions among the elements of a column (for example, combination of different types of Care) then there are even more second-order and higher-order components. Further, there are a very large number of 
fragments of these components also encapsulated in the ontology. The ontology encapsulates the combinatorial complexity of a nation's healthcare policy.

Three illustrative components derived from the ontology are shown in Figure 1. They are:

1. National financial policies on accessibility of preventive care for family. For example, policies to provide financial incentives for families to travel to obtain preventive care.

2. National regulatory policies on cost of wellness for individual-aged. For example, policies to limit the cost of annual check-ups for senior citizens.

3. Provider administration policies on parity of illness-episodic and illness-chronic care for family. For example, policies on satellite clinics in low-income neighbourhoods.

A component, fragment, or element in the ontology may or may not be instantiated in a particular policy. Studying across policies, some of them may be instantiated frequently, some infrequently, and others not at all. We will label the frequently instantiated portions the 'bright' spots, the infrequent ones the light' spots, and the overlooked ones the blind/blank' spots.

The luminosity of each spot is a product of two opposing dynamics. A bright' spot may be so because it is effective and important; it may also be a consequence of habit and herd effect ('more of the same'), irrespective of whether it is effective or important. A light' spot may be so because it is ineffective and unimportant; it may also be a consequence of difficulty of implementing it ('path of least resistance'), irrespective of its potential effectiveness or importance, or its recent emergence in importance. A blind/blank' spot may have been simply overlooked by design or by accident; or, it may be infeasible.

Knowing the 'bright', light', and 'blind/blank' spots in the policies and their antecedent reasons will help develop more systemic and systematic approaches to the challenge of healthcare policies. In the following we present an ontological map of health programs and initiatives in Australia, highlight the 'bright', Iight', and 'blind/blank' spots, and discuss possible reasons for the same. Before presenting the results, we will first describe the method we used for mapping. In the conclusion we will present the potential implications of this program of research and the planned extensions to what is presented here.

The distinguishing feature of the proposed ontology is that it articulates the logic of the system in structured natural English words, phrases, and sentences. It is neither an abstract, philosophical discussion for meta-cognition (imagination) of the system, nor a concrete, syntactically precise formalization of the system for machine cognition. It is between the two extremes - a pragmatic, semantically rich articulation of the system for human cognition.

The ontology itself can be refined by adding subcategories of elements, coarsened by aggregating categories of elements, extended by adding additional dimensions, and reduced by eliminating current dimensions. Thus, it can be adapted to design healthcare policy at different levels of complexity and granularity (Ramaprasad and Syn 2015, Ramaprasad, Syn, and Win 2014, Ramaprasad and Syn 2014a).

\subsection{Validity of the Ontology}

The validity of the ontology will determine how well the ontology captures and represents a national healthcare policy. The common methods focus on induced ontologies at a finer level of detail, greater formalism, and machine readability, but not on deduced ontologies at a higher-level of abstraction such as ours. Hence, we draw on the traditional constructs of validity and assert the ontology's face validity, content validity, semantic validity, systemic validity, and external validity, which are commonly used in social science research (Brennan, Voros, and Brady 2011, Horn and Lee 1989).

We can judge the validity of the ontology of national healthcare policy based on the following questions: How explicit is the conceptualization? How logical is the conceptualization? How specific is the conceptualization? How systematic is the description? How systemic is the 
description? Are the dimensions basic to the domain? Are the taxonomies basic to the dimensions? Are the components basic to the domain? We address these questions below to establish the ontology's validity.

The ontology is a complete and closed description of Australia's national healthcare policy. It is logically derived as described earlier. It is a novel reorganization and representation of traditional bodies of knowledge from multiple disciplines such as public policy, healthcare, and social science. It derives part of its validity from that of the knowledge in the underlying disciplines.

It comprehensively describes a country's healthcare policy using structured natural English understandable to novices and experts alike. It deconstructs the combinatorial complexity of a construct and presents it visually as an easily readable, parsimonious text-table (Tufte 1990). The ontology encapsulates all 30,310 possible components of healthcare policy in a readable form in a single sheet of paper. Thus, its face validity is high.

Each logical component of national healthcare policy derived from the ontology is semantically meaningful as illustrated above; thus, its semantic validity is high. It is easy to ascertain whether a component is instantiated or not. Obversely, it is also easy to ascertain the component that matches an instantiation.

Its dimensions are based on the common bodies of knowledge from underlying disciplines. The taxonomies include the basic categories of knowledge from these disciplines. Hence, the content validity of the dimensions, the taxonomies, and the large number of consequent components is high.

Further, the ontology encapsulates all the possible components of Australia's national healthcare policy. It has high systemic validity.

Lastly, the ontology is also validated through application to the data (programs and initiatives). The application will highlight errors of omission of significant elements and of commission of irrelevant elements in the ontology. These errors are easily corrected - the ontology is easily extensible and reducible - and the validity of the ontology enhanced. In the same vein, most of the ontology has been validated through application to Chile's and India's healthcare policies and learning from the same.

\section{Methods}

We downloaded the list of all health programs and initiatives from The Department of Health's website (Australian Government Department of Health 2016d) in April 2015. A total of 163 programs and initiatives were imported into an Excel spreadsheet together with links to the details. Four of the authors used a spreadsheet tool to map each program/initiative to the ontology. Each coder coded about half the programs. The coding assignment ensured that each coder's programs overlapped about 50\% with a second coder's and the rest 50\% with a third coder's. Thus, each program was coded by at least two coders, and each coder's coding was compared with two others' for validation.

The programs and initiatives were coded in two rounds with significant feedback and learning between the rounds. The coders were instructed to base their judgments on explicit attributes of the programs and initiatives in the description. They were also instructed to resist imputing attributes.

The first round of coding was based on the coders' understanding of the terms based on the project discussions. A comparison of the coding of overlapping sets (as described above) revealed significant variations in the same. The feedback on differences was provided to the coders on a color-coded spreadsheet as well as an ontological map (described later). The feedback revealed to each coder his/ her bias with reference to two other coders.

The feedback was discussed by the coders. Part of the differences was attributed to the absence of glossary of terms. In other words, coders' interpretations of the terms were slightly different 
in some cases. Consequently, a consensus glossary as shown in Appendix was developed, after a few iterations, to aid the second round of coding.

The second round of coding was based on the glossary. The comparison of coding revealed much greater agreement than in the first round. The coders debated whether they should undertake a third round to achieve greater consensus or base subsequent analysis on the union of their coding. The latter was adopted for the following reasons. It would be more inclusivethe final data would include what one might expect to be natural differences in interpretation of the text data. It may not be possible to achieve complete consensus given the nature of the data, the backgrounds of the coders, and the interpretation necessarily involved in coding. Thus, overall, the final coding traded possible errors of omission for possible errors of commission.

The mapping of health programs and initiatives to the ontology is based on the presence or absence of concepts related to individual ontological elements. The coding is symmetric in nature since both presence (coded as ' 1 ') and absence (coded as '0') of an element are significant. The similarity between symmetric variables is generally measured by Simple Matching Coefficient (SMC) (Cheetham and Hazel 1969, Gower 1971). The average SMC of the final coding is $90.63 \%$, a significant agreement among coders.

\section{Results}

We present the results of the analysis as an ontological map (Figure 2) and a dendrogram (Figure 3). The former highlights the emphasis on the elements of the ontology in the 163 programs and initiative. The latter highlights the clustering of elements of the ontology in the corpus. They provide two different but related perspectives on Australia's national health programs and initiatives. We discuss the two in greater detail below.

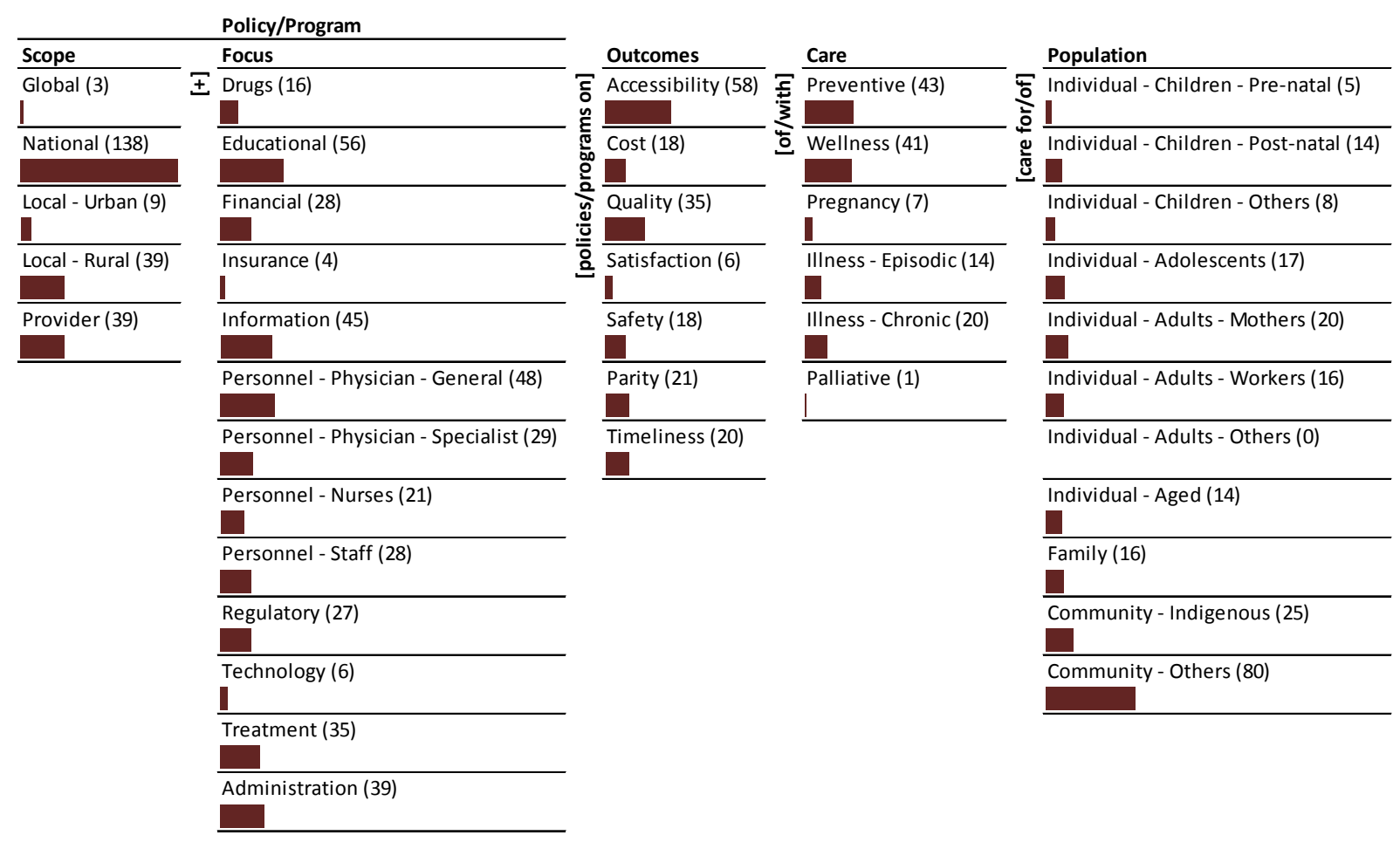

Figure 2 Ontological Map of Monads

\subsection{Ontological Map}

The ontological map of individual ontological elements (monads) is shown in Figure 2. The number in parentheses adjacent to an element indicates its frequency of occurrence in the 163 health programs and initiatives. The data bar below the element is a visual indicator of the same scaled to the maximum frequency of occurrences of any one element, National (138) in 
this case. A program/initiative may be coded on multiple elements on a dimension (column) or no items. Hence, the sum of the numbers in each column may not equal 163-it may bemore or less. The visualization in Figure 2 clearly highlights the areas of emphasis, limited emphasis, and no emphasis. We will discuss the map in detail below from right to left.

\subsection{Population}

The dominant focus of the programs is the Community-Others (80), the general community, and Community-Indigenous (25), the indigenous community. The programs are dominantly community-based, not family- and individual-based.

The secondary focus of the programs is Individual-Adults-Mother (20), IndividualAdolescents (17), Individual-Adults-Workers (16), Family (16), Individual-Aged (14), and Individual-Children-Post-Natal (14).

The tertiary focus is on Individual-Children-Other (8) and Individual-Children-Pre-Natal (5).

It is noted, however, that there are 5 or more programs that address the needs of each segment of the population identified in the ontology. There are no 'blind/blank' spots in the Population dimension.

\subsection{Care}

The dominant focus of the programs is on Preventive (43) and Wellness (41) care. The secondary focus is on Illness-Chronic (20) and Illness-Episodic (14) care. The tertiary focus is on Pregnancy (7). One program focuses on Palliative (1) care. Preventive and wellness care are the dominant drivers of the programs.

Combining the Care and Population dimensions it may be reasonable to infer that the dominant emphasis of the programs is on the preventive/ wellness care of the community.

\subsection{Outcomes}

The dominant outcomes sought are Accessibility (58) and Quality (35). The secondary outcomes are Parity (21), Timeliness (20), Cost (18), and Safety (18). Satisfaction (6) is tertiary. Accessibility followed by quality is the dominant driver of the programs.

Combining the Outcomes, Care, and Population dimensions it may be reasonable to infer that the dominant emphasis of the programs is on the accessibility/ quality of preventive/ wellness care of the community.

\subsection{Focus}

The dominant focus of the programs is Educational (56), Personnel-Physician-General (48), Information (45), Administration (39), and Treatment (35). The secondary focus is on Personnel-Physician-Specialist (29), Financial (28), Personnel-Staff (28), Regulatory (27), and Personnel-Nurses (21). The tertiary focus is on Drugs (16), Technology (6), and Insurance (4).

Combining the Focus, Outcomes, Care, and Population dimensions it may be reasonable to infer that the dominant emphasis of the programs is on education/general physician/ information/administration/treatment policies for accessibility/quality of preventive/ wellness care of the community.

\subsection{Scope}

The dominant scope is National (138) - it is to be expected given that only national programs and initiatives were coded. There are however many programs that are Local-Rural (39) and Provider related. Very few are Local-Urban (9) and Global (1).

Combining all the dimensions it may be reasonable to infer that the dominant emphasis of the programs is on National education/general physician/information/ administration/ treatment policies for accessibility/ quality of preventive/ wellness care of the community. 


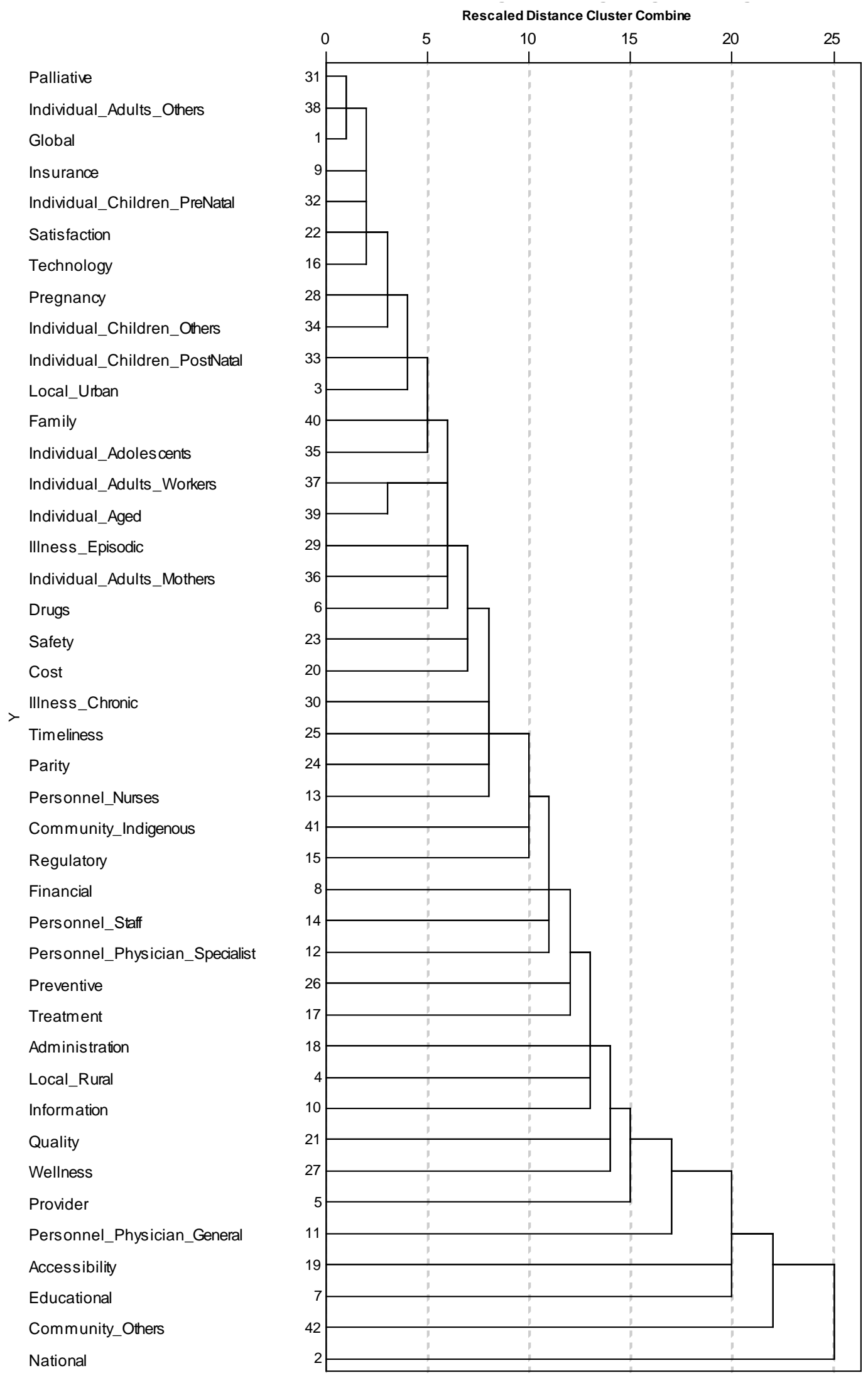

Figure 3 Cluster Analysis of Elements of National Health programs 


\section{Cluster Analysis}

The dominant themes can only be inferred from the ontological map (Figure 2); it cannot be confirmed because it lacks the data on the co-occurrence of the elements in a program. The clusters of ontology elements based on their co-occurrence are shown in Figure 3.

The clusters are formed based on the coding similarity between pairs of ontology elements in the corpus measured by SMC (Cheetham and Hazel 1969, Gower 1971). As mentioned above, SMC is a symmetric similarity measure which considers presence (coded as ' 1 ') and absence (coded as ' 0 ') of elements in the articles, in contrast to other well-known binary similarity/ distance measures such as Jaccard and Sørensen-Dice which only consider presence of elements. In ontological analysis both presence and absence of elements in articles convey equally important information. If for example two elements are not mapped to any of the articles, both J accard and Sørensen-Dice methods will return a value of zero to indicate a complete dissimilarity whereas SMC will return a value of one to indicate a complete agreement which is the appropriate representation for the ontological analysis. In cases where articles include or exclude two elements in no particular pattern, all three similarity measures are comparable. However, the denominator in Jaccard and Sørensen-Dice formulas is a variable based on the number of articles coded to one or both of a pair of elements. Thus, the denominator can vary from one pair to another. The denominator in SMC formula is a fixed number that represents the total number of programs and initiatives coded. SMC will thus provide a more consistent comparison across pairs of elements. Further, the cluster analysis is used to descriptively summarize the data about the population of articles and not to make statistical inference about the population from a sample of programs and initiatives.

The clustering was conducted in SPSS based on the Single Linkage (or Nearest Neighbour) between the clusters. The single linkage method, as the name implies, links cluster elements based on the nearest neighbour in the existing clusters. The computation in this method is simple, and consequently the agglomeration coefficients of and the dendrogram from the cluster analysis can be translated to the data and interpreted as presented, as discussed below. (This is not the case with the Centroid method, for example.) As such the method provides a direct visualization of the association between the ontology elements. These associations can be interpreted in conjunction with the frequencies of the elements in the ontological map (Figure 2).

The dendrogram in Figure 3 visually depicts the matching of the ontology elements in the corpus of programs based on the SMC. At the top are the two most infrequent elements in the ontological map-Palliative (1) and Individual-Adults-Others (0). Their SMC (0.994) reflects their matched absence in 162 of the 163 programs, with no matched presence. At the bottom are the two most frequent elements in the ontological map-National (138) and CommunityOthers (80). Based on their SMC (0.607) one can compute that they have matched presence in 77 programs and matched absence in 22 programs. In the rest (66) one of them is present. One may compute similar values for each pair of joined elements based on their frequency of occurrence (from the ontological map) and their SMC (from the dendrogram).

It may be observed in the dendrogram that: (a) the less frequently present elements in the ontological map are at the top and the more frequently present elements are at the bottom, and (b) the adjacent elements are more closely matched than distant ones. By using the five equidistant divisions of SMC we can infer the following themes in descending order of dominance in the programs corpus. Considering more divisions will result in finer-grained themes, fewer divisions will result in coarser-grained themes. They are:

1. National programs/policies for care of community-others. This is the dominant theme of the corpus.

2. Personnel-Physician-General and educational policies/programs for accessibility of care. 
3. Local-Rural/ Provider

information/administration/ treatment/financial/ personnel-physician-

specialist/personnel-staff policies/programs for quality of preventive/ wellness care.

4. Drugs/ Regulatory/ Personnel-Nurses

policies/ programs

for parity/timeliness/cost/ safety of illness-chronic/illness-episodic care for/of individual-adults-mothers/ individual-adults-workers/individualaged/community-indigenous care.

5. Global/Local-Urban technology/ insurance policies/ programs for satisfaction with pregnancy/palliative care for individual-adolescents/individual-children-postnatal/individual-adults-others/ family care.

The first two may be said to be the dominant themes of the corpus. The next two are nondominant themes. The last is really a non-theme rather than a theme, highlighting what is virtually absent from the corpus. In the following section we will discuss the themes and nontheme inferred from the ontological map and cluster analysis of Australia's national health programs.

\section{Discussion}

National accessibility of healthcare for the community emerges as the core theme of Australia's health programs and initiatives, based on the ontological map and the dendrogram. Further, within Cluster 1, Accessibility and the general Community are very closely associated. Accessibility is explicitly articulated in the Department of Health's Strategic Priorities. Logically, it is a precondition for all other outcomes. Its reflection in the country's programs and initiatives signifies the effective translation of the priorities into programs and initiatives. On the other hand, community-based healthcare is articulated only for "[r]egulation that protects the health and safety of the community" (Australian Government Department of Health 2016c) as a strategic priority. The emphasis on community in the programs appears to be broader than that envisaged in the strategic priorities.

The theme of the second cluster may be parsed by studying the underlying sub-clusters. The bottom-most sub-cluster of eight elements highlights the emphasis on the timeliness of care of individuals and families. The next sub-cluster of four elements highlights the emphasis on information and treatment for preventive and wellness care of the individuals and families. These are aligned with the strategic priorities of "[a]n integrated approach that balances prevention, primary, secondary and tertiary care" and "[p]romoting greater engagement of individuals in their health and healthcare" (Australian Government Department of Health 2016c).

The complex theme of the third cluster may be best understood as a combination of two distinct sub-themes. The bottom sub-cluster focuses on provider educational/drug programs for cost/ parity both episodic and chronic illness care in indigenous communities. The second subcluster focuses on personnel and financial programs for rural care. These are aligned with the strategic priority of "[e]nabling access for the most disadvantaged including Aboriginal and Torres Strait Islander people, people in rural and remote areas and people experiencing socioeconomic disadvantage" (Australian Government Department of Health 2016a).

The fourth cluster consists of the residual elements. There does not appear to be an underlying theme. However, we note the following dyads. Palliative Care is grouped with IndividualAdults-Others to form a dyad of elements virtually absent in the programs. The same is true of Global scope and Insurance programs. Pregnancy care is paired with Individual-ChildrenPrenatal-a logical association, but infrequent among the programs. By the same token, the association of Regulatory and Administration programs is understandable-they are part of many programs. Last, Local-Urban is paired with Safety and then linked to Quality. It is interesting to note that Quality is not strongly associated with the care elements. It is, at the next level, associated with the Regulatory-Administration dyad. It may be because quality is 
seen as an administrativeand regulatory issue rather than as a care issue in the programs. Part of this may be the realization of the strategic priority of "[a]ffordable, accessible, efficient, and high quality health system through... [r] egulation that protects the health and safety of the community" (Australian Government Department of Health 2016c). However, in the same cluster Technology is associated with Satisfaction-both occur infrequently in the programs. Logically, Technology programs can affect satisfaction but that doesn't appear to be a strong sub-theme in the programs. It is contrary to the strategic priority of "[b]etter, more costeffective patient care through innovation and technology" (Australian Government Department of Health 2016c). Moreover, without an emphasis on Technology (and innovation) it would be difficult to achieve the overall purpose to "[l]ead and shape Australia's health system" (Australian Government Department of Health 2016c) in today's healthcare environment.

In summary, Australia's Health Programs and Initiatives map well to its Vision, Purpose, and Strategic Priorities. There are no normative benchmarks on the relative emphasis on the different elements of the ontology and the clustering of these elements. It may be impossible and inappropriate to emphasize all elements equally. Moreover, some of the elements may be emphasized in the programs of the states, territories, regional, and local areas. For example, regional programs are likely to have dominant rural focus in contrast to the national programs. Yet, the ontological map and the clusters also reveal several light' spots in the corpus which may limit the programs' overall effectiveness. The ontological map and the dendrogram provide a 'big picture' of the programs for further reflection. We suggest a few based on the above analysis and discussion.

Accessibility to healthcare is necessary but not sufficient to achieve the vision of the Department of Health. It is the first step but subsequently the other outcomes-cost, quality, satisfaction, safety, parity, and timeliness-assume greater importance. Without these, accessibility by itself may be of little value. Initiatives for providing accessibility of healthcare in rural areas include "Rural Clinical Training support", "Mental Health Services in Rural and Remote Area", and "Bonded Medical Services Scheme". Some programs such as the "Primary Health Network", ATAPS "Access to Allied Psychological Services", "National partnership agreement indigenous early childhood development program", although national, have a rural and regional scope.

Further, the limited emphasis on technology programs may also need reflection. Today, technology has become sine qua non for realizing all the outcomes. It would also be appropriate to prioritize it at the national level to facilitate interoperability, standardization, etc.

In the same vein, there has been some emphasis on aged care. The "National Palliative Care Program" emphasizes palliative care. As the demographics change and chronic illnesses increase, more palliative care programs may become necessary. Focus on preventive and curative care alone, as in the past, may not be sufficient for the healthcare system.

In the next section, we conclude with a discussion of the contributions of this paper, some of its assumptions and limitations, and plan for future research.

\section{Conclusion}

Traditional health policy analyses often consider the outcome of the policies from a particular stakeholder's perspective (policy makers, doctors, allied staff etc.), in a narrow domain (communicable diseases, maternal and child health, etc.), or a geographical area (rural, urban, etc.). A key contribution of the present analysis presented is that it provides a unified view of all these various perspectives and foci. The highest level of the ontology provides a unification of concepts corresponding to these multiple perspectives of analyses: Scope, Focus, Outcomes, Care, and Population. The use of an ontology as a knowledge sharing mechanism is not new. However, the use of an ontology to enable combining and integrating the analysis of policies is new and is an innovative approach to gain a broader and a deeper understanding of theimpact of health policies regardless of their scope or the stakeholders involved. 
Ideally research should find application in programs; programs should provide feedback and pose problems for research; and policy should follow research and programs, and provide feedback for both. In this context we believe that the ontology can help guide, inform, and direct the research, programs, and policies on national healthcare systemically and systematically instead of fragmentarily and opportunistically. The ontology can be used to develop a roadmap for future research on national healthcare policy.

1. The paper makes three basic contributions to understanding comprehensive assessment and direction of national health programs. They are:

1. An approach using an ontology based on national healthcare policy. The approach can be extended, refined, revised, and adapted to other contexts (countries, states, territories, cities, etc.);

2. A method of mapping the state-of-the-programs for national healthcare using the ontology based on text data about the programs; and

3. A method of analysing the dominant, less dominant and non-dominant focus of a nation's programs, and the consequent gaps in them.

There is a need for policy makers to understand the implications of the above findings for future planning, prioritizing for formulation (and reformulation) of policy. Our research will allow the policy makers to ask, as discussed above, for example: Are the dominant foci e.g., preventative care, accessibility balanced against the low focus elements such as palliative care, satisfaction, and technology? Do they need to be? Ultimately ontological analysis such as this can provide a pragmatic basis for recommendations by policy makers and interest groups for policy formulation. Future work will investigate broadening the effectiveness of the approach to provide detailed input that can be of used to reflect on federal budget allocation across the programs and initiatives. This will require enhancing the coding with numeric values to reflect the "degree of emphasis" in monetary terms.

In ending the discussion we should also highlight some of the limitations of the research. Despite our best efforts the ontology may be incomplete or over-specified. We are reasonably confident, based on the study of the corpus while coding, that the errors of omission and commission are minimal. In the future, should it be necessary, the ontology can be extended, reduced, refined, or coarsened as appropriate.

The results are based on the population of Australia's national health programs at a point in time. There may be state and local programs which may be complementary and relevant. In the future, the domain could be systematically broadened to be more inclusive.

Considerable effort has been expended in the construction of the glossary and the monitoring of coding to minimize errors. While the coders tried to stay true to the text of the programs without imputing their own expectations, one cannot exclude the possibility of over-coding and under-coding. Given the large population of programs (163) and the significant variation in the frequency of the elements, despite the potential errors, the results are likely to be robust.

Given the data, errors in the ontological map and dendrogram are unlikely. However, there is room for variation in the interpretation of the luminosity of the different elements and the determination of clusters. Since the method of construction of the map and dendrogram are completely transparent, it would be easy to compare and contrast different interpretations of the same.

In summary, despite the limitations, the insights are strong. Their explanation as to why the corpus is as described may vary, but there is little room for variation in the description of the programs corpus. 


\section{References}

Australian Government Department of Health. 2016a. "Annual Reports." Accessed February 11, 2016. http:// www.health.gov.au/internet/main/ publishing.nsf/Content/Annual+Reports-3.

Australian Government Department of Health. 2016b. "History of the Department." Accessed February 2016. http:// www.health.gov.au/internet/ main/publishing.nsf/Content/ health-history.htm.

Australian Government Department of Health. 2016c. "Our Vision." Accessed February 11, 2016. http:// www.health.gov.au/ internet/ main/ publishing.nsf/Content/ annual-report-1415toc 14-15-our-vision.

Australian Government Department of Health. 2016d. "Programs \& Initiatives." Accessed $\quad$ February 2016. http:// www.health.gov.au/ internet/ main/ publishing.nsf/Content/ programsinitiatives-menu.

Bennett, Sara, and David H. Peters. 2015. "Assessing National Health Systems: Why and How." Health Systems and Reform 1(1):9-17. doi: 10.1080/23288604.2014.997107.

Bourke, Lisa, J ohn S. Humphreys, J ohn Wakerman, and J udy Taylor. 2012. "Understanding rural and remote health: A framework for analysis in Australia." Health and Place 18 (3):496-503. doi: 10.1016/j.healthplace.2012.02.009.

Brennan, Linda, J oseph Voros, and Erica Brady. 2011. "Paradigms at play and implications for validity in social marketing research." J ournal of Social Marketing 1 (2):100-119. doi: 10.1108/20426761111141869.

Cheetham, Alan H., and Joseph E. Hazel. 1969. "Binary (Presence-Absence) Similarity Coefficients." J ournal of Paleontology 43 (5):1130-1136. doi: 10.2307/ 1302424.

Churchman, C. West. 1967. "Wicked Problems." Management Science 14 (4): B-141-42.

Cimino, J ames J . 2006. "In defense of the Desiderata." J ournal of Biomedical Informatics 39 (3):299-306. doi: 10.1016/j.jbi.2005.11.008.

Fisher, Matthew, Frances E. Baum, Colin MacDougall, Lareen Newman, and Dennis McDermott. 2016. "To what Extent do Australian Health Policy Documents address Social Determinants of Health and Health Equity?" J ournal of Social Policy 45 (03):545-564. doi: 10.1017/ S0047279415000756.

Gill, G. K., and H. Babacan. 2012. "Developing a cultural responsiveness framework in healthcare systems: an Australian example." Diversity and Equality in Health and Care 9 (1):45-55.

Gower, J . C. 1971. "A General Coefficient of Similarity and Some of Its Properties." Biometrics 27 (4):857-871. doi: 10.2307/ 2528823.

Gruber, Thomas R. 1995. "Toward Principles for the Design of Ontologies Used for Knowledge Sharing." International J ournal Human-Computer Studies 43 (5-6):907-928. doi: 10.1006/ijhc.1995.1081.

Gruber, Thomas R. 2008. "Ontology." In Encyclopedia of Database Systems, edited by Ling Liu and M. Tamer Özsu. New York: Springer-Verlag.

Heilbrunn-Lang, Adina Y., Lauren M. Carpenter, Seona M. Powell, Susan L. Kearney, Deborah Cole, and Andrea M. De Silva. 2016. "Reviewing public policy for promoting population oral health in Victoria, Australia (2007-12)." Australian Health Review 40 (1):19-26. doi: 10.1071/ AH15013. 
Hoeffner, Lori Ann Rung, and Richard P. Smiraglia. 2013. "Visualizing Domain Coherence: Social Informatics as a Case Study." Advances in Classification Research Online 23 (1):49-51. doi: 10.7152/ acro.v23i1.14261.

Horn, B. Ray, and In-Hee Lee. 1989. "Toward integrated interdisciplinary information and communication sciences: a general systems perspective." In Proceedings of the 22nd Hawaii International Conference on System Sciences (HICSS 1989), 244-255. KailuaKona, Hawaii, USA: IEEE.

Kazimierczak, Karolina A., Zoë C. Skea, Mary Dixon-Woods, Vikki A. Entwistle, Deb FeldmanStewart, James M. O. N'Dow, and Sara J. MacLennan. 2012. "Provision of cancer information as a "support for navigating the knowledge landscape": Findings from a critical interpretive literature synthesis." European J ournal of Oncology Nursing 17:110. doi: 10.1016/j.ejon.2012.10.002.

Kickbusch, Ilona, Warren McCann, and Tony Sherbon. 2008. "Adelaiderevisited: from healthy public policy to Health in All Policies." Health Promotion International 23 (1):1-4. doi: 10.1093/ heapro/ dan006.

Koh, Howard K., Julie J . Piotrowski, Shiriki Kumanyika, and J onathan E. Fielding. 2011. "Healthy People: A 2020 Vision for the Social Determinants Approach." Health Education \&Behavior 38 (6):551-557. doi: 10.1177/ 1090198111428646.

Larkins, Sarah, and Rebecca Evans. 2014. "Greater support for generalism in rural and regional Australia." Australian Family Physician 43 (7):487-490.

Lopes, Mário Amorim, Álvaro Santos Almeida, and Bernardo Almada-Lobo. 2015. "Handling healthcare workforce planning with care: where do we stand?" Human Resources for Health 13 (1):1-19. doi: 10.1186/ s12960-015-0028-0.

Malatzky, Christina, and Lisa Bourke. 2016. "Re-producing rural health: Challenging dominant discourses and the manifestation of power." J ournal of Rural Studies 45: 157164. doi: 10.1016/j.jrurstud.2016.03.005.

Moore, Michael, and Heather Yeatman. 2014. "'Killer Budget' attacks prevention and primary health care." Australian and New Zealand J ournal of Public Health 38 (4):301. doi: 10.1111/ 1753-6405.12276.

Noar, Seth M., and Rick S. Zimmerman. 2005. "Health Behavior Theory and cumulative knowledge regarding health behaviors: are we moving in the right direction?" Health Education Research 20 (3):275-290. doi: 10.1093/ her/ cyg113.

Núñez Mondaca, Alicia, Arkalgud Ramaprasad, and Thant Syn. 2015. "National Healthcare Policies in Chile: An Ontological Meta-Analysis." In MEDINFO 2015: eHealth-enabled Health, edited by Indra Neil Sarkar, Andrew Georgiou and Paulo Mazzoncini de Azevedo Marques, 1105.

Quine, Willard Van Orman. 1961. From a Logical Point of View. Second, revised ed. Boston, MA, USA: Harvard University Press.

Ramaprasad, Arkalgud, B. Sastry Nanda Kumar, and Thant Syn. 2015. "National Healthcare Programs and Policies in India: An Ontological Analysis." In Proceedings of the 10th Annual International Conference on Public Policy and Management (10 CPPM 2015). Bangalore, India.

Ramaprasad, Arkalgud, and Thant Syn. 2014a. "Design Thinking and Evaluation Using an Ontology." In Design Science: Perspectives from Europe, edited by Markus Helfert, Brian Donnellan and Jim Kenneally, 63-74. Switzerland: Springer International Publishing.

Ramaprasad, Arkalgud, and Thant Syn. 2014b. "Ontological Topography: Mapping the Bright, Light, Blind/Blank Spots in Healthcare Knowledge." In Proceedings of the 2nd 
International Conference on Big Data and Analytics in Healthcare (BDAH 2014). Singapore.

Ramaprasad, Arkalgud, and Thant Syn. 2015. "Ontological Meta-Analysis and Synthesis." Communications of the Association for Information Systems 37:138-153.

Ramaprasad, Arkalgud, Thant Syn, and Mohanraj Thirumalai. 2015. "Ontological Analysis of Meaningful Use of Healthcare Information Systems (MUHIS) Requirements and Practice." In Biomedical Engineering Systems and Technologies, edited by Guy Plantier, Tanja Schulz, Ana Fred and Hugo Gamboa, 315-330. Switzerland: Springer International Publishing.

Ramaprasad, Arkalgud, Thant Syn, and Khin Than Win. 2014. "Ontological Meta-Analysis and Synthesis of HIPAA." In Proceedings of the 18th Pacific Asia Conference on Information Systems (PACIS 2014). Chengdu, China.

Scharnhorst, Andrea. 2001. "Constructing Knowledge Landscapes Within the Framework of Geometrically Oriented Evolutionary Theories." In Integrative Systems Approaches to Natural Social Dynamics, edited by M. Mathies, H. Malchow and J. Kriz, 505-515. Springer Berlin Heidelberg.

Seeleman, Conny, Marie-Louise Essink-Bot, Karien Stronks, and David Ingleby. 2015. "How should health service organizations respond to diversity? A content analysis of six approaches." BMC Health Services Research 15 (1). doi: 10.1186/s12913-015-1159-7.

Smith, James A., Gemma Crawford, and Louise Signal. 2016. "The case of national health promotion policy in Australia: Where to now?" Health Promotion J ournal of Australia 27 (1):61-65. doi: 10.1071/HE15055.

Syed, Kamran, Mark Kröll, Vedran Sabol, Arno Scharl, Stefan Gindl, Michael Granitzer, and Albert Weichselbraun. 2012. "Dynamic Topography Information Landscapes - An Incremental Approach to Visual Knowledge Discovery." In Data Warehousing and Knowledge Discovery, edited by Alfredo Cuzzocrea and Umeshwar Dayal, 352-363. Springer Berlin Heidelberg.

Tate, Mary, Elfi Furtmueller, J oerg Evermann, and Wasana Bandara. 2015. "Introduction to the Special Issue: The Literature Review in Information Systems." Communications of the Association for Information Systems 37:103-111.

Tufte, Edward R. 1990. Envisioining Information. Cheshire, CT: Graphics Press.

World Health Organization. "Quality of Care." Last Modified September 5, 2016. http:// www.who.int/ management/ quality/assurance/QualityCare_B.Def.pdf.

World Health Organization. 2011. "World Health Organization Health Systems Strengthening Glossary." Accessed February 6, 2016. http:// www.who.int/ healthsystems/Glossary_J anuary2011.pdf.

Zhang, J uan, J un Xie, Wanli Hou, Xiaochen Tu, Jing Xu, Fujian Song, Zhihong Wang, and Zuxun Lu. 2012. "Mapping the Knowledge Structure of Research on Patient Adherence: Knowledge Domain Visualization Based Co-Word Analysis and Social Network Analysis." PLoS ONE 7 (4). doi: 10.1371/journal.pone.0034497. 


\section{Appendix: Glossary}

Policy: Healthcare policy.

Scope: Reach of the healthcare policy.

Global: Policy applicable in all countries of the world.

National: Policy applicable everywhere in Australia.

Local: Policy applicable within a defined part of Australia.

Urban: Policy applicable within local urban areas.

Rural: Policy applicable within local rural areas.

Provider: Policy applicable to a healthcare providing institution.

Focus: Focus of the healthcare policy.

Drugs: Policies regarding drugs used in healthcare.

Educational: Policies regarding healthcare education.

Financial: Policies regarding healthcare finance.

Insurance: Policies regarding healthcare insurance.

Information: Policies regarding healthcare information.

Personnel: Policies regarding healthcare personnel.

Physician: Policies regarding physicians.

General: Policies regarding general physicians.

Specialist: Policies regarding specialist physicians.

Nurses: Policies regarding healthcare nurses.

Staff: Policies regarding healthcare staff.

Regulatory: Policies regulating healthcare.

Technology: Policies regarding healthcare technology.

Treatment: Policies regarding healthcare treatment.

Administration: Policies regarding healthcare administration.

Outcomes: The intended outcomes of healthcare policy.

Accessibility: The accessibility of healthcare to the population.

Cost: The cost of healthcare to the population.

Quality: The quality of healthcare delivered to the population.

Satisfaction: The population's satisfaction with healthcare.

Safety: The safety of healthcare delivered to the population.

Parity: The parity of healthcare delivered to the population segments.

Timeliness: The timeliness of healthcare delivery to the population.

Care: The different types of healthcare.

Preventive: Care to prevent illnesses and diseases in the population.

Wellness: Care to maintain wellness of the population.

Pregnancy: Care during pregnancy.

Illness: Care of illnesses when they occur.

Episodic: Care during illness episodes -- time bound.

Chronic: Care of chronic illnesses -- continuing.

Palliative: Care to alleviate pain and suffering.

Population: The population targeted by the policy.

Individual: Individual recipients of healthcare.

Children: Children who are recipients of healthcare.

Pre-natal: Unborn children receiving care during pregnancy.

Post-natal: Newborn children receiving care.

Other: Children beyond post-natal care, pre-adolescent.

Adolescents: Adolescents receiving healthcare.

Adults: Adults receiving healthcare.

Mothers: Mothers receiving maternal healthcare.

Workers: Workers receiving occupational/work related healthcare. 
Other: Adults other than mothers and workers. Aged: Older people receiving elderly healthcare.

Family: Family, as an entity, receiving healthcare.

Community: Community as an entity receiving healthcare. Indigenous: Healthcare of the indigenous populations.

Other: Healthcare of other identified communities.

Copyright: (c) 2016 Ramaprasad, Win, Syn, Beydoun, Dawson. This is an open-access article distributed under the terms of the Creative Commons Attribution-NonCommercial 3.0 Australia License, which permits non-commercial use, distribution, and reproduction in any medium, provided the original author and AJ IS are credited.

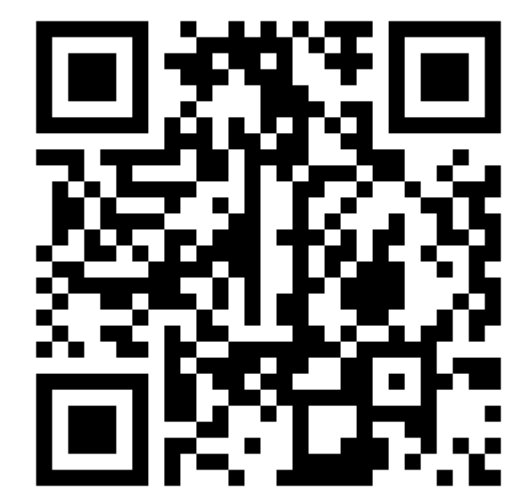

